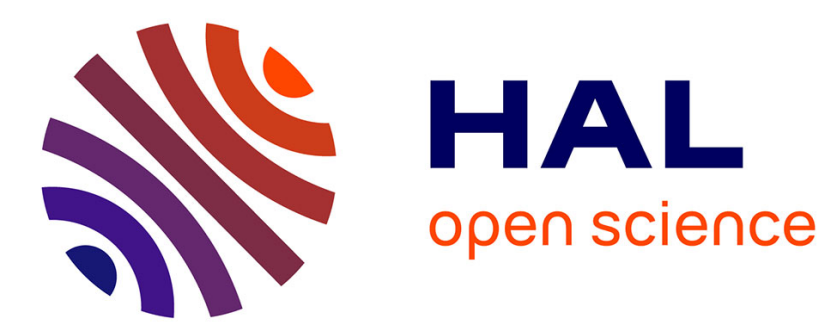

\title{
Software defined networking (SDN) for reliable user connectivity in 5G networks
}

Siwar Ben Hadj Said, Bernard Cousin, Samer Lahoud

\section{To cite this version:}

Siwar Ben Hadj Said, Bernard Cousin, Samer Lahoud. Software defined networking (SDN) for reliable user connectivity in 5G networks. Network Softwarization (NetSoft), 2017 IEEE Conference on, Jul 2017, Bologna, Italy. 10.1109/NETSOFT.2017.8004219 hal-01660873

\section{HAL Id: hal-01660873 https://hal.science/hal-01660873}

Submitted on 14 Dec 2017

HAL is a multi-disciplinary open access archive for the deposit and dissemination of scientific research documents, whether they are published or not. The documents may come from teaching and research institutions in France or abroad, or from public or private research centers.
L'archive ouverte pluridisciplinaire HAL, est destinée au dépôt et à la diffusion de documents scientifiques de niveau recherche, publiés ou non, émanant des établissements d'enseignement et de recherche français ou étrangers, des laboratoires publics ou privés. 


\section{Software Defined Networking (SDN) for reliable user connectivity in 5G Networks}

\author{
Siwar Ben Hadj Said \\ CEA, LIST, Communication Systems Laboratory \\ 91190 Gif-sur-Yvette, FRANCE \\ firstname.lastname@cea.fr
}

\author{
Bernard Cousin, Samer Lahoud \\ Université de Rennes I, IRISA \\ 35042 RENNES, FRANCE \\ \{firstname.lastname\}@irisa.fr
}

\begin{abstract}
Reliability was, and still is, a major concern for telecoms operators. Network outage can potentially produce high penalty in terms of revenue and user quality of experience. In this paper, we advocate that the data plane reliability can be easily improved when the Software Defined Networking (SDN) concept is incorporated into LTE/EPC networks. As a way to ensure user connectivity be available even in case of network equipment failures, we propose two recovery modes: (i) automatic tunnel re-establishment and (ii) on-demand tunnel re-establishment. Through simulations, we show that those mechanisms outperform the recovery mechanism previously proposed in 3GPP standards. Index Terms-connectivity, LTE, recovery, SDN, OpenFlow.
\end{abstract}

\section{INTRODUCTION}

In $5 \mathrm{G}$ vision, network operators are invited to rethink their architectures in order to meet the myriad of possible use cases, user requirements and business opportunities. Indeed, new technologies, enable the increase in radio access networks capacity. However, the higher transmission rates provided by new radio interfaces put a strain on the access and core networks to support a great number of applications.

Reliable connectivity is among the most crucial design keys of $5 \mathrm{G}$ networks. We define the connectivity as the set of network mechanisms that ensures the proper delivery of user data packets (e.g. ciphering, mobility tunnels, header compression, etc.). In current Long Term Evolution / Evolved Packet Core (LTE/EPC) networks, any network equipment failure may cause tremendous strain on mobile operators and may lead to a temporary service outage [1]. Indeed, due to a network equipment failure, all active connections using that specific equipment are interrupted. Consequently, the user quality of experience may be degraded. Moreover, the reestablishment of impacted sessions may generate a significant amount of delay and signaling as new connectivity must be established for each impacted session. This may ultimately, cause total network breakdown.

Software Defined Networking (SDN) is a recent trend in communications networking, whereby the behavior of network equipments is controlled by a logically centralized controller. This trend is reshaping the way networks are designed, managed, and secured. In fact, SDN replaces manual and specific interfaces of network equipments with a programmable and open interfaces. This enables the automation of tasks such as network equipment configuration and traffic policy management [2]. Therefore, SDN may enable network operators to manage connectivity service according to various trigger criteria, at a given time (defining and using their own policy). This approach allows the evolution from current static to highly dynamic network deployments, where the network topology, configuration, and dimensioning can change over time depending on various contextual information.

With the upcoming of SDN-based network architectures, ensuring reliable connectivity is becoming more and more feasible. A reliable connectivity can be defined as the ability of the data session to recover quickly and smoothly from certain types of failure or overload situations and yet remain functional from the subscriber perspective. In fact, in SDNbased network architecture, the network control functions are decoupled from the data forwarding plane. Therefore, a failure in data plane may not impact the control functions. These latter, when supported with the adequate mechanisms, can rapidly recover the impacted data sessions and achieve, therefore, reliable connectivity.

In [1], we proposed a new design for LTE/EPC network where the data plane reliability can be achieved. In this paper, we extend the proposed design and propose two recovery mechanisms to ensure reliable connectivity in data plane.

The remainder of this paper is as follows. Section II provides an overview of the related work. Then, Section III presents, in details, the SDN-based LTE/EPC architecture. Section IV proposes the main recovery mechanisms to ensure reliability in LTE/EPC network. Section V explains how our proposed mechanisms enable a faster recovery than what was proposed in 3GPP specifications. Section VI uses bandwidth utilization and packet loss metrics to evaluate the proposed recovery mechanisms through simulations. Section VII concludes the paper.

\section{RELATED WORK}

Reliability was, and still is, one of the key design features of network architectures. For instance, [3] proposes an improved gateway failure restoration mechanism for better resiliency in UMTS networks. [4] uses analytical models to analyze the reliability of UMTS networks at different levels. They showed that by incorporating fault tolerance mechanisms in UMTS networks can achieve substantial gains in network reliability. In LTE/EPC networks, a major concern was dedicated to the resiliency of base stations [5] [6]. The 3GPP technical 
specification [7] proposes some basic restoration mechanisms for gateway failures. However these mechanisms are not sufficient as they automatically interrupt sessions and impacts the subscriber QoE significantly [1].

Introducing network programmability feature to mobile networks has been the focus of recent research works [8] [9]. [10] addresses the flexibility to be gained in a cellular network when the SDN concept is applied. It has completely reshaped the LTE/EPC architecture by replacing gateways with OF switches, middleboxes and a logically centralized controller. The reliability of their architecture has not been studied. [11] addresses network resiliency in $5 \mathrm{G}$ networks. It proposes a self-healing framework for networks where SDN concepts are incorporated. Their framework is destined to manage faults in different planes (i.e. data, control, application and service planes). They gave some examples of faults and the possible recovery actions. However, they did not present in details how these actions can be achieved in the network.

\section{OVERVIEW OF SDN-BASED LTE/EPC ARCHITECTURE}

In this section, we extend the proposed architecture in [1] by separating the control functions from the data forwarding function in SGWs and PGWs of the same pool area as shown in Figure 1. As a result, the whole intelligence of the control plane in the SGW (i.e. SGW-C software) and PGW (i.e. PGW$\mathrm{C}$ software) are logically centralized and runs on top of the OF Controller (OF-ctrl) as an application. The data plane functions are performed by the SGW data plane (SGW-D) and PGW data plane (PGW-D). Also, the MME software runs on top of the OF-ctrl as an application.

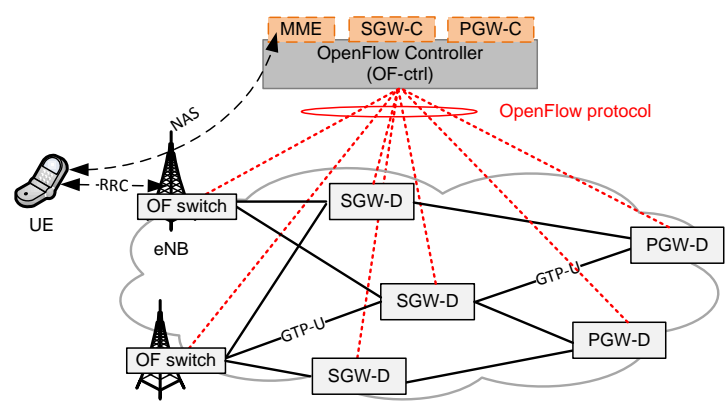

Fig. 1: SDN-based LTE/EPC architecture.

The control plane is composed of the following entities:

- OpenFlow Controller (OF-ctrl): it manages the forwarding data plane between eNB, SGW-D and PGW-D.

- $\boldsymbol{M M E}$ : it is responsible for UE authentication and authorization, and mobility management.

- S-GW control plane (SGW-C): it represents the SGW intelligence part. It participates in GTP tunnel establishment by allocating Tunnel Endpoint IDentifier (TEID). The SGW-C allocates unique TEID value per session for the uplink traffic within the S1-U interface. It allocates also unique TEID value for the downlink traffic within S5-U interface.
- P-GW control plane (PGW-C): it represents the PGW intelligence part. It participate in GTP tunnel establishment by allocating TEID that will be used in the uplink traffic within S5-U interface. The PGW-C is responsible for IP address allocation. In addition, it decides the QoS requirement and administrates the traffic shaping, gating control, rate enforcement, and bearer binding in the PGW$\mathrm{D}$ accordingly.

The data plane is composed of the following entities:

- eNB: it keeps the same radio functions specified by 3GPP standards (e.g. scheduling, radio resource management, etc.). A support for OF protocol is added to eNB in order to enable data plane configuration by the OF-ctrl.

- SGW data plane (SGW-D): it serves as an advanced OF switch that is able to encapsulate/decapsulate GTP packets. It is responsible for packet forwarding between the eNB and PGW-D.

- PGW data plane (PGW-D): it serves as an advanced OF switch that supports GTP encapsulation/decapsulation. It is responsible for packet forwarding and QoS actions enforcement in the data plane.

\section{How to handle failures in Data Plane}

Recovery mechanisms are crucial for a reliable connectivity in network architectures. The resilience of the proposed architecture depends on fault-tolerance in the data forwarding plane (i.e. node redundancy as eNB, SGW-D or PGW-D) and on the high availability of the logically centralized control functions (i.e. OF-ctrl, MME, SGW-C and PGW-C). In the proposed architecture, equipment failure in the data plane can be easily handled. As the OF-ctrl exchanges periodic Echo Request/Reply messages with equipments in the data plane, it can detect equipment failures rapidly.

In this section, we propose two recovery mechanisms that enable the controller to handle an SGW-D failure. The same approach is applied to PGW-D failures. Regarding the eNB failures, the same mechanisms are applied when the UE is under the coverage of an available neighboring eNB.

Upon an SGW-D failure, the controller can behave in different manners depending on the impacted session requirements. For example, if the impacted session is sensitive to delays such as sessions related to live streaming application, the controller should rapidly re-establish the connectivity (i.e. GTP tunnel). For this category of sessions, we propose a rapid recovery mode called Automatic connectivity re-establishment.

If the impacted sessions are tolerant to delays (e.g. applications that need connectivity to download asynchronous data such as Facebook application), the controller may re-establish the connectivity on-demand, i.e. only when the UE needs to send/receive data. We call this second recovery mode as $\mathrm{On}$ demand connectivity re-establishment. In the following, we present both of these recovery modes.

\section{A. Automatic connectivity re-establishment}

When the recovery mechanism is in automatic connectivity re-establishment mode, upon detecting a SGW-D failure, the 
associated SGW-C selects a new one (i.e. SGW-D 2) for the impacted sessions. After that, the OF-ctrl updates the entries in eNB and PGW-D with the IP address of the new SGWD by sending OFPT_MODIFY_STATE messages. Then, the connectivity in the data plane is re-established as shown in Figure 2. The double arrow between UE and eNB and the cylinders between eNB, SGW-D and PDW-D represent the user connectivity in the data plane.

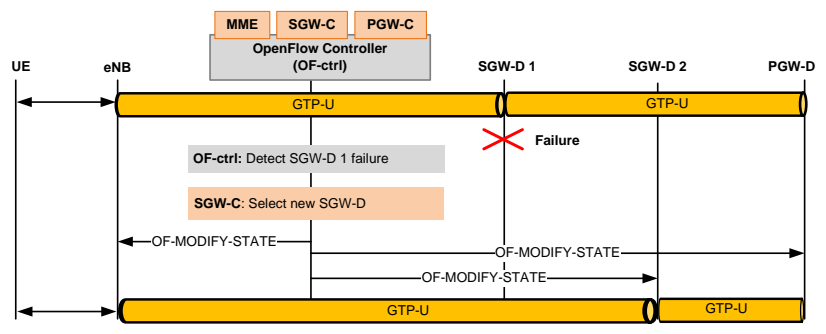

Fig. 2: Automatic connectivity re-establishment.

Here, we can see the advantage of logical centralizing the TEID allocation function related to SGWs. Indeed, the SGW-C does not create new TEID values during the recovery mechanism. The OF-ctrl updates just flow entries in eNB and PGW-D with the new SGW-D IP address. However, the OFctrl inserts a new flow entry in SGW-D 2 to enable traffic forwarding between eNB and PGW-D.

\section{B. On-demand connectivity re-establishment}

When the recovery mechanism is in On-demand connectivity re-establishment mode, upon detecting a SGW$D$ failure, the associated SGW-C removes entries in eNB and PGW-D related to the impacted sessions by sending OFPT_DELETE_STATE messages. In the meantime, the SGW-C may anticipate the upcoming packets of each impacted session by selecting new SGW-D. The anticipated path may be memorized in the controller. The OF-ctrl will not enforce the new path in the data plane until it receives a new data packet related to the impacted session (i.e. a data packet from the UE that arrives at the eNB or a data packet destined to the UE that arrives at the PGW-D). For instance, when the UE had data traffic to send, it requests from the eNB a data radio bearer. Then, it sends the data traffic to the eNB. This latter includes the header of the first data packet received from the UE in the OFPT_PACKET_IN message and sends it to the OF-ctrl. After that, the OF-ctrl determines the tuple (address source, address destination, port source, port destination) from this packet and checks its data base for the anticipated path. The OF_MODIFY_STATE messages are used to setup the user connectivity in the data plane.

The OF-ctrl does not command the removal of the radio data bearer between the UE and the eNB. In fact, in the radio part of the $\mathrm{eNB}$, a release timer is always triggered whenever the UE enters IDLE state. At timer expiration, the radio data bearer will be removed. Therefore, in this recovery mechanism, the radio access bearer establishment procedure is run when the radio data bearer is inactive.

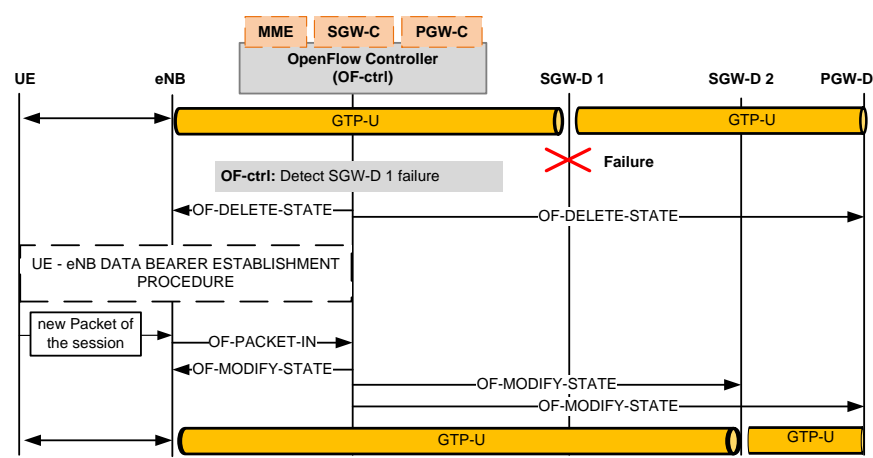

Fig. 3: On-demand connectivity re-establishment.

This mode is similar to what is proposed today in 3GPP standards as it does not re-establish the connectivity immediately. However, this mechanism differs from 3GPP standards in that new paths may be anticipated to enable a fast recovery of the connectivity for the new upcoming packets related to impacted sessions.

\section{RECOVERY MODES MODELING AND ANALYSIS}

In this section, we model the proposed recovery mechanisms and compare them to $3 \mathrm{GPP}$ recovery mechanism. We define the recovery period as the time required to re-establish tunnels upon a failure. To analyze this parameter in details, we consider the following parameters:

$T d$ : detection time (i.e. the MME/OF-ctrl detects that an SGW/SGW-D failure occurs)

$T n$ : notification time (i.e. the MME/OF-ctrl notifies the eNB and PGW/PGW-D to delete flow entries related to the impacted sessions)

$T e$ : tunnel establishment time (i.e. the MME triggers the tunnel establishment between the eNB, SGW and PGW). This procedure includes the radio bearer establishment, and the whole exchanges between eNB, MME, SGW and PGW in order to establish the GTP tunnels.

$T r$ : tunnel re-establishment time (i.e. the SGW-C selects a new SGW-D and triggers the OF-ctrl to configure it). It includes the data path update and eventually the radio bearer establishment. In general, it is smaller than the tunnel establishment time ( $\mathrm{Te}$ ).

\section{A. Recovery mechanisms in SDN-based LTE/EPC architecture}

Figure 4 models the time to recovery (TTR) of the proposed recovery mechanisms in SDN-based LTE/EPC architecture.

In the automatic connectivity re-establishment mode, the recovery procedure is composed of a detection phase $(T d)$, a notification phase $(T n)$, and tunnel re-establishment phase $(T r)$. Therefore, the TTR is calculated as follows:

$$
T T R^{\text {Automatic }}=T_{d}+T_{n}+T_{r}
$$

Similarly to the automatic connectivity re-establishment procedure, the on-demand recovery procedure includes the detection time $(T d)$, the notification phase $(T n)$ and the reestablishment phase $(T r)$. However, in this procedure, the 
controller should wait till the next data packet of the UE to re-establish the tunnel (i.e. $T_{\text {wait }}$ ). Thus, the TTR of this procedure is calculated as follows:

$$
T T R^{\text {OnDemand }}=T_{d}+T_{n}+T_{\text {wait }}+T_{r}
$$

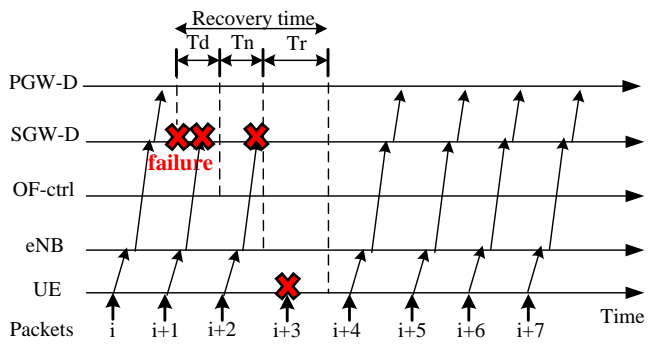

(a) Automatic connectivity re-establishment

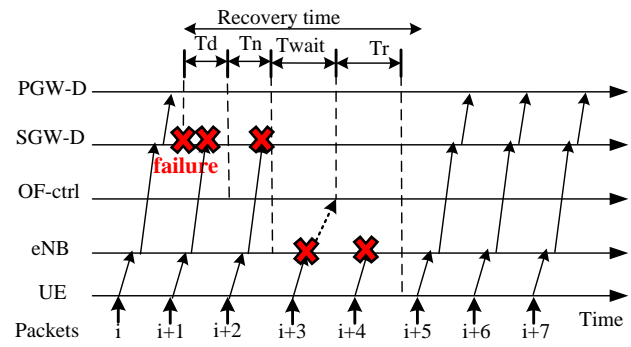

(b) On-demand connectivity re-establishment

Fig. 4: Timing diagram of SDN-based recovery mechanisms.

\section{B. Recovery mechanism in 3GPP LTE/EPC architecture}

Figure 5 models the recovery period according to the $3 \mathrm{GPP}$ recovery mechanism. It consists in 4 main phases. After a detection phase $(T d)$, the MME should notify eNB $(T n)$. Then, the MME waits the arrival of the next connectivity request from the UE to establish a new GTP tunnels $\left(T_{\text {wait }}\right)$. Upon receiving a service request, the MME triggers the connectivity establishment procedure $\left(T_{e}\right)$. The TTR is calculated as follows.

$$
T T R^{3 G P P}=T_{d}+T_{n}+T_{w a i t}+T_{e}
$$

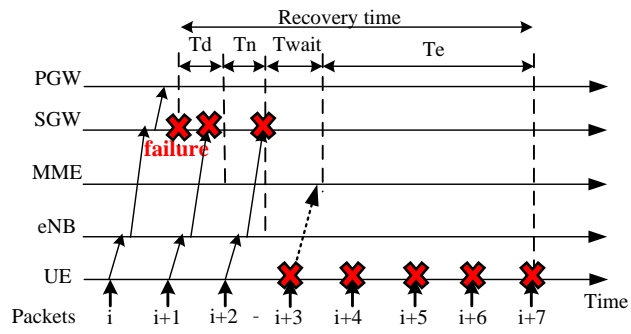

Fig. 5: Timing diagram of 3GPP recovery mechanism.

\section{Vi. Performances Evaluation}

\section{A. Performance metrics}

The European report [12] presents various metrics to measure network resilience to faults. In our study, we focus on the following metrics:
1) Bandwidth utilization: It represents the throughput measured by a system within a specified period. When the network encounters failures, a low variation of bandwidth indicates a high level of resilience.

2) Packet loss: It represents the percentage of packets that are lost in transit from source to destination, during the equipment failure. Packet loss is an indicator for network resilience when it is experiencing failures. A low packet loss indicates a high resilience against faults.

\section{B. Metrics evaluation}

The simulation setting can be briefly described as follows. The simulations are conducted on the topology shown in Figure 6. Our topology is composed of 11 OF switches. S1 and S11 act as the eNB and PGW-D, respectively. S5, S6 and $\mathrm{S} 7$ act as SGW-Ds. The role of the remaining switches is to ensure connectivity between eNB, SGW-Ds and PGW-D. The host $\mathrm{H} 1$ represents the UE in our simulation. The host $\mathrm{H} 2$ acts as, for instance, a server in the Internet. We used the emulator Mininet to create the desired topology. We choose the POX controller as a base for the OF-ctrl module. The proposed recovery mechanisms are implemented in POX. To simulate data traffic between $\mathrm{H} 2$ to $\mathrm{H} 1$, we used the Iperf tool.

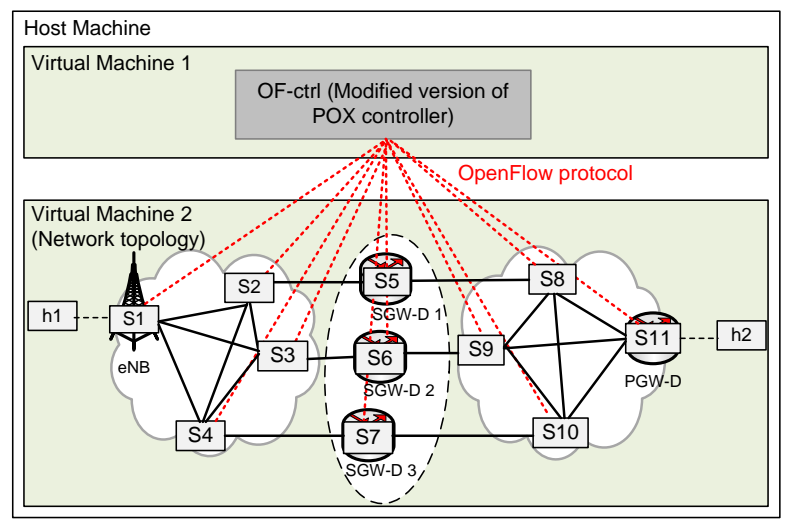

Fig. 6: Test topology in Mininet.

In our simulated topology, the link between UE (i.e h1) and eNB is characterized with a delay of $5 \mathrm{~ms}$ and a bitrate of $100 \mathrm{Mbits} / \mathrm{s}$ and each link between OF switches has a delay of $5 \mathrm{~ms}$ and a bitrate of $1000 \mathrm{Mbits} / \mathrm{s}$ [13] [14]. According to [15] and [16], the GTP tunnel establishment takes around $500 \mathrm{~ms}$. In addition, notifying the eNB about the SGW failure takes around $7.5 \mathrm{~ms}$. To determine whether the SGW is alive, the MME and SGW exchange echo request and reply messages. The SGW failure detection time value is evaluated to 90 seconds in standard 3GPP [17].

To simulate an SGW failure in our simulation, we simply shutdown one of the SGW (i.e. S5, S6, or S7) in mininet. In the first experiment, we set up a TCP connection between the UE (i.e. H1) and the server (i.e H2). Then, after $7 s$, we manually shutdown the SGW participating in data path. The TCP throughput is shown in Figure 7. We note that, our proposal is more resilient to failures in data plane than $3 \mathrm{GPP}$ network. In fact, we note that the TCP throughput is recovered 
to the best value after only $2 s$ with our recovery mechanisms. However, with 3GPP recovery mechanism, the TCP session is interrupted. This can be explained by different facts: the MME waits too much time to declare an SGW/SGW-D failure, it deletes any context related to the impacted GTP tunnels, it waits till the UE tests the effective connectivity, and it launches the full connectivity establishment procedure.

After that, we simulated a UDP traffic with a uniform distribution, a bitrate of $1 \mathrm{MBit} / \mathrm{s}$ and during $20 \mathrm{~s}$. The 3GPP standard recovery mechanism leads to around $53 \%$ of packet loss, whereas the automatic and on-demand recovery mechanisms achieve $0.35 \%$ and $0.37 \%$. As we can see, the 3GPP recovery mechanism incurs the highest packet loss compared to our recovery mechanisms. In fact, with the SDN-based LTE/EPC architecture, the SGW failure is rapidly detected compared to the $3 \mathrm{GPP}$ architecture. This is due to the use of TCP sockets in the communication between control and data plane. In 3GPP standard architecture, the communication between equipments is based on GTP control protocol (GTPC) which uses UDP. To detect an equipment failure, an echo request/reply process is implemented. For instance, MME will wait $90 s$ for an echo reply before declaring an SGW failure. To get an idea about the detection time impact on the packet loss, we varied the detection time $\left(T_{d}\right)$ parameter between $10 \mathrm{~s}$ and $150 \mathrm{~s}$. For each value, we generate a UDP traffic with a uniform distribution, a bitrate of $1 \mathrm{MBits} / \mathrm{s}$ and a duration of $250 \mathrm{~s}$. The packet loss percentages in 3GPP scenario are depicted in Figure 8. As we can see, the packet loss percentage increases when the detection time increases.

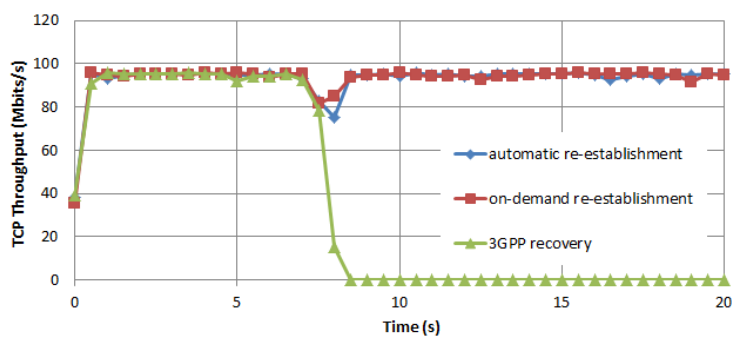

Fig. 7: TCP throughput under SGW failure.

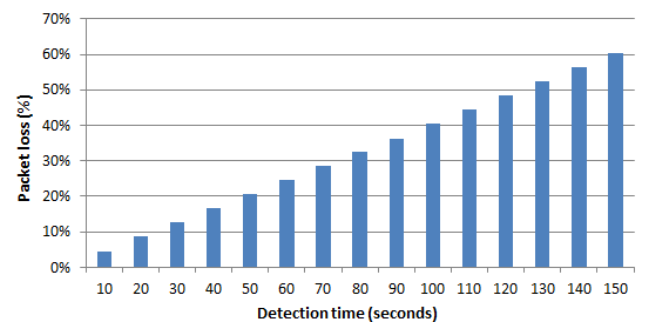

Fig. 8: Packet loss in 3GPP scenario.

\section{CONCLUSION}

In this paper, we proposed two recovery mechanisms to ensure the connectivity resiliency towards any network equipment failure in $5 \mathrm{G}$ networks. We showed, through simulations, that the SDN adoption in 5G networks augmented by the proposed recovery mechanisms outperforms the $3 \mathrm{GPP}$ LTE/EPC architecture in terms of throughput and packet loss in case of a network equipment failure in data plane. In future work, it is interesting to evaluate the connectivity availability of the proposed recovery modes using analytic studies. Then, an implementation of the proposed mechanisms in a real testbed will give insights about the Time To Repair (TTR) of the connectivity in both recovery modes.

\section{ACKNOWLEDGMENT}

The work presented in this paper is supported by the FrenchGerman project BERCOM jointly funded by ANR and BMBF under the grant number ANR-14-PICS-0001.

\section{REFERENCES}

[1] S. B. H. Said, M. R. Sama, K. Guillouard, L. Suciu, G. Simon, X. Lagrange, and J. M. Bonnin, "New control plane in 3gpp lte/epc architecture for on-demand connectivity service," in IEEE 2nd International Conference on Cloud Networking (CloudNet), Nov 2013, pp. 205-209.

[2] Open Networking Foundation (ONF), "Software-Defined Networking: The New Norm for Networks," Tech. Rep., 2012.

[3] P. Lin and G.-H. Tu, "An improved ggsn failure restoration mechanism for umts," Wireless Networks, vol. 12, no. 1, pp. 91-103, 2006.

[4] S. Dharmaraja, V. Jindal, and U. Varshney, "Reliability and survivability analysis for umts networks: An analytical approach," Network and Service Management, IEEE Transactions on, vol. 5, no. 3, pp. 132-142, 2008.

[5] D. Griffith, R. Rouil, A. Izquierdo, and N. Golmie, "Measuring the resiliency of cellular base station deployments," in Wireless Communications and Networking Conference (WCNC), 2015 IEEE. IEEE, 2015, pp. 1625-1630.

[6] H. Farooq, M. S. Parwez, and A. Imran, "Continuous time markov chain based reliability analysis for future cellular networks," in 2015 IEEE Global Communications Conference (GLOBECOM). IEEE, 2015, pp. $1-6$.

[7] 3GPP, "Restoration procedures," 3rd Generation Partnership Project (3GPP), TS 23.007, 2012.

[8] D. Kreutz, F. M. Ramos, P. Verissimo, C. E. Rothenberg, S. Azodolmolky, and S. Uhlig, "Software-defined networking: A comprehensive survey," proceedings of the IEEE, vol. 103, no. 1, pp. 14-76, 2015.

[9] B. A. Nunes, M. Mendonca, X.-N. Nguyen, K. Obraczka, and T. Turletti, "A survey of software-defined networking: Past, present, and future of programmable networks," Communications Surveys \& Tutorials, IEEE, vol. 16, no. 3, pp. 1617-1634, 2014.

[10] Softcell: Scalable and flexible cellular core network architecture. ACM, 2013.

[11] J. Sánchez, B. Yahia, I. Grida, N. Crespi, T. Rasheed, and D. Siracusa, "Softwarized $5 \mathrm{~g}$ networks resiliency with self-healing," in $5 G$ for Ubiquitous Connectivity (5GU), 2014 1st International Conference on. IEEE, 2014, pp. 229-233.

[12] European Network and Information Security Agency (ENISA), "Measurement Frameworks and Metrics for Resilient Networks and Services: Technical Report," https://www.enisa.europa.eu/ activities/Resilience-and-CIIP/Incidents-reporting/metrics/reports/ metrics-tech-report/at_download/fullReport, Tech. Rep., 2011.

[13] A. Larmo, M. Lindstrom, M. Meyer, G. Pelletier, J. Torsner, and H. Wiemann, "The lte link-layer design," Communications Magazine, IEEE, vol. 47, no. 4, pp. 52-59, 2009.

[14] Nokia Networks white paper, "Technology Vision 2020 Reducing network latency to milliseconds," Tech. Rep., 2015.

[15] Z. Savic, "Lte design and deployment strategies," http: //www.cisco.com/web/ME/expo2011/saudiarabia/pdfs/LTE_Design_ and_Deployment_Strategies-Zeljko_Savic.pdf, CISCO, 2012.

[16] 3GPP, "Requirements for further advancements for Evolved Universal Terrestrial Radio Access (E-UTRA)," 3rd Generation Partnership Project (3GPP), TR 36.913, 2013.

[17] Juniper, "echo-interval," http://www.juniper.net/techpubs/en US/junos-mobility11.2/topics/reference/configuration-statement/ echo-interval-edit-unified-edge-gateways-ggsn-pgw.html, 2011. 